\title{
Dynamic Characteristics Design System for 3D Nanostructure Fabricated by FIB-CVD*
}

\author{
Shunjiro NISHI ${ }^{* *}$, Reo KOMETANI ${ }^{* *}$, Shinichi WARISAWA ${ }^{* *}$ \\ and Sunao ISHIHARA** \\ **Department of Mechanical Engineering, School of Engineering, The University of Tokyo, \\ 7-3-1 Hongo, Bunkyo, Tokyo 113-8685, Japan \\ E-mail: syun_west@nanome.t.u-tokyo.ac.jp
}

\begin{abstract}
Small-sized structures of diamond-like carbon (DLC) deposited by FIB-CVD contain microscopic non-uniformity in their mechanical properties. This is mainly due to the structures containing $\mathrm{Ga}$ implanted by $\mathrm{Ga}$ ion irradiation during deposition [1]. In the application of DLC nanostructures to mechanical devices, the non-uniform properties cause difficulties in designing nanomechanical devices. To establish a modeling method for nanomechanical device design, the precise mechanical properties of DLC pillars were evaluated. For the property characterization, several techniques, such as transmission electron microscopy, ion implantation simulation, and density measurement by vibration, were examined. Then, an analysis model for finite element method-based design of dynamic characteristics was developed.
\end{abstract}

Key words: Focused Ion Beam, FIB-CVD, Resonator, Modeling, FEM-based Simulation, 3D Nanostructure

\section{Introduction}

Focused-ion-beam chemical vapor deposition (FIB-CVD) is a key technology for fabricating nanomechanical structures because FIB-CVD enables us to fabricate arbitrary three-dimensional (3D) nanostructures with various materials such as diamond-like carbon (DLC) and tungsten [1,3]. The microscopic material properties of fabricated structures, however, are not uniform because Ga is implanted during $\mathrm{Ga}^{+} \mathrm{FIB}$ irradiation [2], and this non-uniformity causes difficulties in designing the functions or performance of nanomechanical devices. To solve this problem, development of a dynamic characteristics evaluation system for 3D nanostructures is necessary. We thus experimentally analyzed the properties of DLC fabricated by FIB-CVD.

Because the material properties are radially inhomogeneous, the material characteristics of a DLC pillar fabricated by the FIB-CVD system SMI9200 (SII Nano Technology Inc.) were evaluated using transmission electron microscope (TEM) techniques, Monte Carlo method-based ion implantation simulation, and density measurement using the vibration method. An ion beam profile was estimated through etching experiments and Gaussian fitting. The Ga distribution in DLC pillars was calculated by Monte Carlo simulation. The density was obtained through measuring the resonance frequency shift of a self-detection-type micro cantilever by thin film deposition. The Young's Modulus distribution in DLC pillars was also estimated using resonance frequency measurement by means of the electron beam irradiation method [4].

Second, we developed an analysis model for finite element method (FEM)-based evaluation of dynamic characteristics using the Ga distribution, DLC density, and Young's Modulus data obtained from the measurement results mentioned above.

[DOl: 10.1299/jamdsm. 4 (No.8] 10-0078)

Copyright $(C 2010$ by JSME 


\section{Measurement and Characterization}

\subsection{Ion beam profile}

The radial flux distribution of a FIB incident to a plane surface can be well described by a Gaussian function:

$$
I(r, \sigma)=\frac{I_{0}}{2 \pi \sigma^{2}} \exp \left(-\frac{r^{2}}{2 \sigma^{2}}\right)
$$

where $I(r, \sigma)$ is the current density, $r$ is the radial distance, $\sigma$ is the standard deviation, and $I_{0}$ is the beam current. $I_{0}$ can be measured using a Faraday cup [5]. For a beam spot size $d$, a full width at half maximum (FWHM) can be expressed as follows:

$$
d=2 \sqrt{2 \ln 2} \sigma .
$$

To determine $\sigma$, a single line etching experiment on a Si substrate was carried out with a $30 \mathrm{keV}$ focused $\mathrm{Ga}^{+}$ion beam and $1.5 \mathrm{pA}$ beam current.

Figure 1 shows the atomic force microscope (AFM) micrograph of line structures for a scanning speed of $1.96 \mu \mathrm{m} / \mathrm{s}$. Typical section analysis was performed at lines a-e. An average of the results is shown in Fig. 2. From the analytical fitting with the Gaussian function, the standard deviation $\sigma$. was measured to be $23.6 \mathrm{~nm}$ and the beam spot size $d$ was calculated to be $55.6 \mathrm{~nm}$ by Eq. (2). From these results, the beam profile of the focused $\mathrm{Ga}^{+}$ion beam was estimated as shown in Fig. 3.

\subsection{Distribution of Ga in DLC pillar}

To develop the analysis model of DLC pillars for mechanical property characterization, the Ga distribution in a pillar must be examined. First, the radial distribution of Ga ions implanted into a pillar was calculated using a Monte Carlo method-based ion implantation

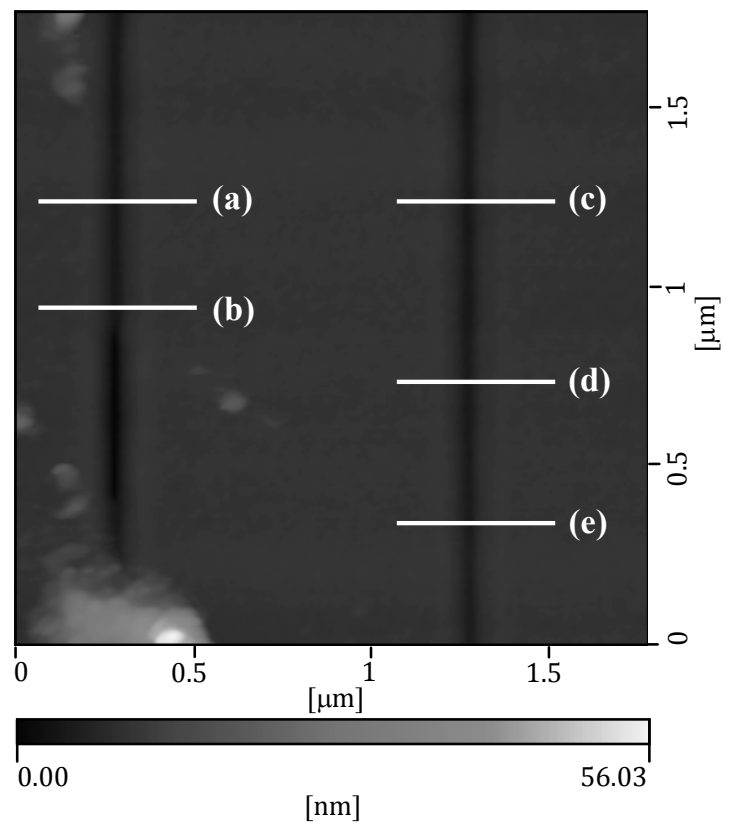

Fig. 1 Atomic force microscope (AFM) micrograph of etching lines and section analysis lines (a-e). 


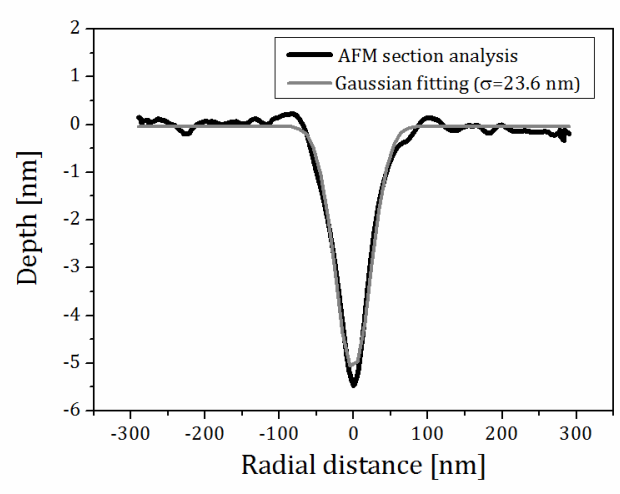

Fig. 2 Average of section analysis and analytical fit function (Gaussian)

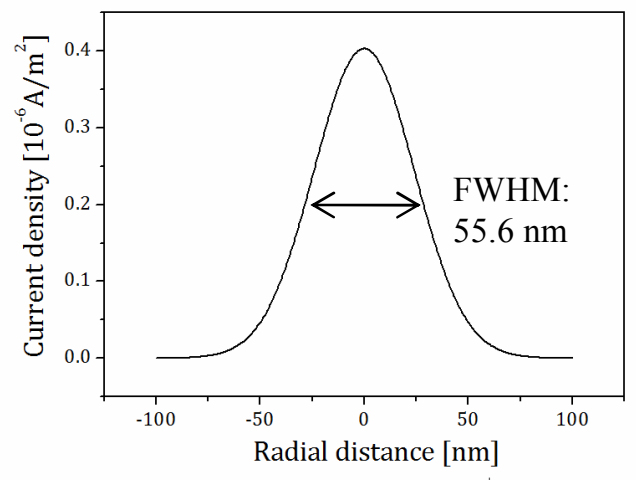

Fig. 3 Beam profile of focused $\mathrm{Ga}^{+}$ion beam

simulation, where the simulation software SRIM (the Stopping and Range of Ions in Matter [6]) and the beam profile shown in Fig. 3 were used.

The radial distribution of the $\mathrm{Ga}^{+}$ions that penetrated the DLC pillar is shown in Fig. 4. FWHM was $57.4 \mathrm{~nm}$, which was $1.8 \mathrm{~nm}$ larger than that of the beam profile. The scattering length of the recoil atoms was about $10 \mathrm{~nm}$ outside of the Ga-implanted area. This width is strongly related to the thickness of the carbon shell of the DLC pillar. This is because the carbon shell is an exterior layer of Ga-free amorphous carbon. The carbon shell is formed by the recoil atoms and the secondary electrons scattered in the pass of Ga implantation. The scattering length of $10 \mathrm{~nm}$ agrees well with the thickness of the carbon shell that was shown in the previous study [7].

The distribution of Ga in a DLC pillar was analyzed by using TEM-EDX, which is a useful method for high-resolution observation and analysis of the material composition in a microscopic area. We used TEM-EDX to verify the accuracy of the Ga distribution calculated by SRIM. Fig. 5 is the TEM image of a DLC pillar that was fabricated by FIB-CVD with a $30 \mathrm{keV}$ focused $\mathrm{Ga}^{+}$ion beam and $1.47 \mathrm{pA}$ beam current.

The center part of the pillar is darker compared with the periphery. This difference of brightness is not only due to the geometric thickness but also to the influence of the abundance of $\mathrm{Ga}$ atoms contained in the center of the pillar. The electron beam is absorbed more in the center of the pillar because the mass-absorption coefficient of Ga is larger than that of carbon.

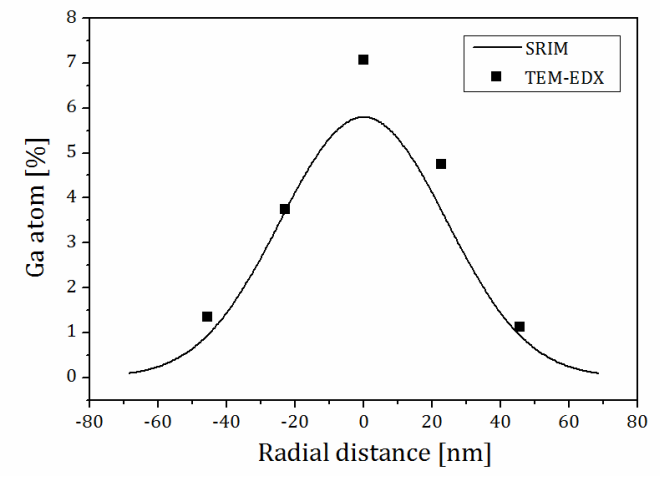

Fig. 4 Distribution of Ga in DLC pillar: result of $\mathrm{Ga}^{+}$ion beam implantation simulation using SRIM, and measurement results by using TEM-EDX

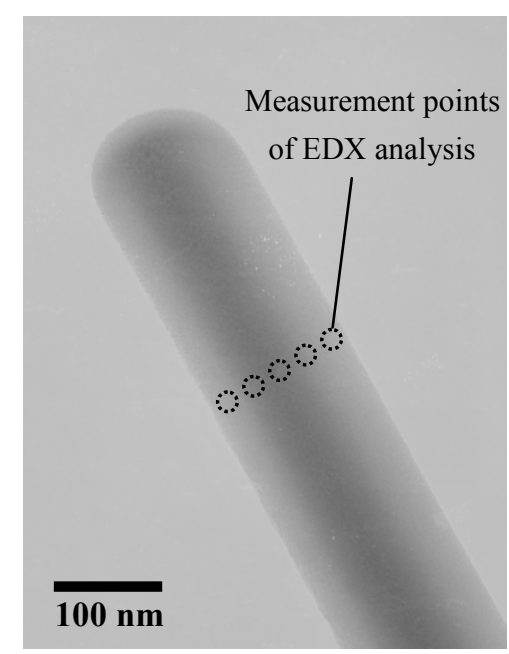

Fig. 5 TEM image of DLC pillar on Mo mesh, and measurement points of EDX analysis 
To examine the Ga distribution in more detail, EDX analysis was performed. The measurement points of the EDX analysis are shown in Fig. 5. The measurement results of EDX are shown in Fig. 4 with the results of SRIM simulation. The analyzed results for both measurement and simulation agree well with each other.

\subsection{Density measurement}

To develop the density distribution model using the Gaussian function, the standard deviation of the Ga distribution in the pillar calculated with SRIM could be applied. In addition, it was still necessary to experimentally evaluate the maximum and minimum values. The minimum value of density (at the carbon shell) was approximated by that of graphite in this study.

The maximum value of density was evaluated using the frequency shift of a self-detecting cantilever (SII-SS-ML-PRC120, NPX1CTP004 SII Nano Technology Inc.). It is very hard to measure the density of the DLC pillar directly because the mass of the DLC pillar is too light compared with the cantilever. Therefore, we measured the density of DLC thin films assuming that their densities were nearly equivalent to that of the center part of a DLC pillar. This is because DLC thin film contains a large amount of Ga, as much as the center part of a DLC pillar. When a thin film is deposited on the cantilever, its resonance frequency decreases according to the mass adhesion. The change in mass in relation to the change in resonant frequency can be given as follows:

$$
\Delta f=\frac{2 \pi^{2} f_{0}^{3}}{k} \Delta m
$$

where $\Delta m$ is the change in mass, $k$ is a spring constant, $f_{0}$ is the initial resonance frequency, and $\Delta f$ is the shift in resonance frequency.

This equation describes the single-degree-of-freedom vibration system. Estimating the mass $\Delta m$ is very difficult due to its smallness and position on the lever, so we cannot calculate the density analytically by using Eq. (3). Therefore, the density was calculated by using FEM-based dynamic characteristic simulation (ANSYS 11.0 Academic Teaching Advanced).

After the DLC thin film had been deposited on the cantilever, its shape was measured by AFM. The measurement results are shown in Fig. 6. A square-shaped DLC thin film was deposited with a $74.19 \mathrm{pA}$ beam current, and the resonance frequency was decreased from 300.194 down to $299.200 \mathrm{kHz}$. The shape and size of the film was modeled on the cantilever in ANSYS, and the density was calculated from the shift of the resonance frequency. Since the density might be influenced by the beam current during deposition, we measured the beam current dependency of its density. The results are shown in Table 1. The difference of the beam current had no effect. By this result, the density of the center part of the DLC pillar was estimated to be $4.8 \mathrm{~g} / \mathrm{cm}^{3}$.

Table 1. Measurement results; dependency of beam current on density of DLC thin films

\begin{tabular}{cccc}
\hline \hline $\begin{array}{c}\text { Beam current } \\
{[\mathrm{pA}]}\end{array}$ & $\begin{array}{c}\text { Frequency shift } \\
{[\mathrm{Hz}]}\end{array}$ & $\begin{array}{c}\text { Deposition volume } \\
{\left[\mu \mathrm{m}^{3}\right]}\end{array}$ & $\begin{array}{c}\text { Density } \\
{\left[\mathrm{g} / \mathrm{cm}^{3}\right]}\end{array}$ \\
\hline 74.19 & 994 & 57.5 & 4.8 \\
20.52 & 606 & 30.7 & 4.7 \\
5.46 & 338 & 14.5 & 4.8 \\
\hline \hline
\end{tabular}




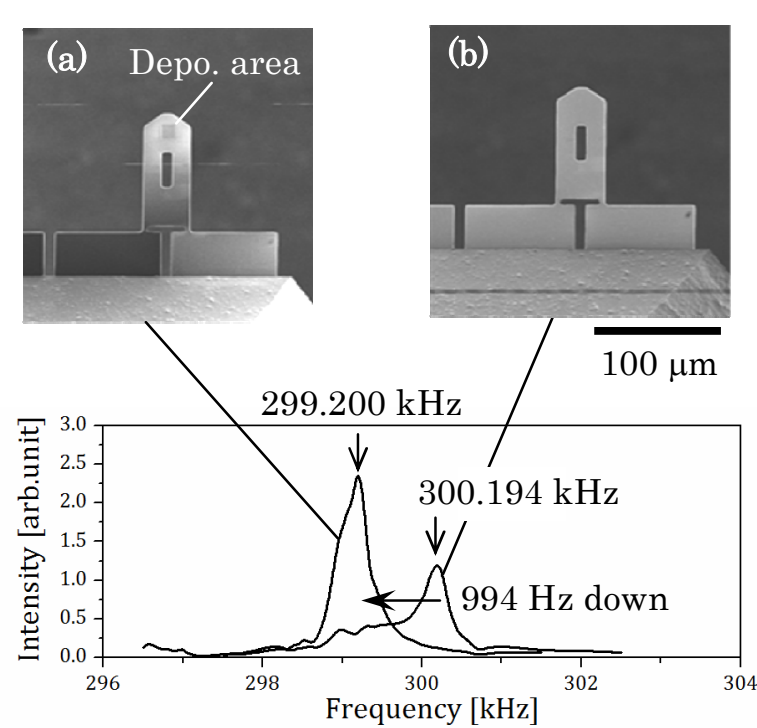

Fig. 6 Frequency shift of self-detecting cantilever; beam current: $74.19 \mathrm{pA}$; volume of DLC: $57.5 \mu^{3}$ (density: $4.8 \mathrm{~g} / \mathrm{cm}^{3}$ ). Scanning ion microscope (SIM) images of cantilever: (a) after deposition, (b) before deposition

\subsection{Young's Modulus modeling}

To develop the distribution model of the Young's Modulus in a DLC pillar using the Gaussian function, the standard deviation, maximum value, and minimum value of the Gaussian are needed as parameters. FWHM of the Gaussian was assumed to be equal to that of the distribution of $\mathrm{Ga}$ in a pillar. The maximum and the minimum values of the Young's Modulus in the model were estimated by the following methods.

When a pillar has a larger width, the resonance characteristics of the pillar are more greatly affected by the material properties of the center of the pillar because of its volume ratio. A material properties distribution model that can be applied to pillars of various widths should be uniquely determined. Therefore, pillars of various widths were fabricated as shown in Fig. 7. Their resonance frequencies were measured by using an electron beam [4]. We determined the maximum and minimum values of the Young's Modulus that satisfied the measured resonance frequency of each pillar width by using FEM-based dynamic characteristics simulation. The maximum value of $375 \mathrm{GPa}$ and minimum value of 13.4 GPa were obtained.
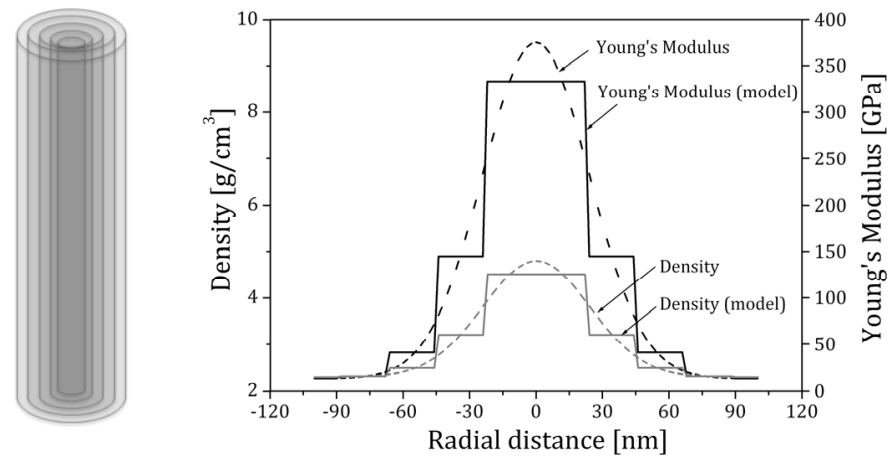

Fig. 8 Pattern diagram of multi-layer model of DLC pillar and material characteristics values given in each layer 


\section{Estimation Model for FEM Simulation}

In accordance with these measurements, the mechanical property distribution model of the DLC pillar was designed as shown in Fig. 8. A multi-layer structural model is suggested. In general, when the number of layers is increased, a longer analytical time will be needed because the size of the mesh will be finer and the number of layer elements increases. It is necessary to select an appropriate number of layers in consideration of the shape of the object and the required accuracy.

To evaluate the performance of the proposed multi-layer model, the two types of nanoresonators shown in Fig. 9 were designed and their properties were evaluated using the model. One resonator was like a long thin plate that broadened to its end, and the other was like a zigzagging pillar. Comparisons between the experimental and analytical results including both multi-layer and single-layer models are shown in Table 2. In the case of the single-layer model, uniform material characteristics were used (Young's Modulus: 187 GPa; density: $\left.3.8 \mathrm{~g} / \mathrm{cm}^{3}[8]\right)$.

The estimation errors of resonance frequencies using multi-layer models were 20.1 and $26.6 \%$ for each type, respectively. On the other hand, the errors in the case of single-layer models were 39.0 and $60.3 \%$, respectively. As shown, the accuracy of the analysis was increased by using the multi-layer model, that is, a property distribution model.
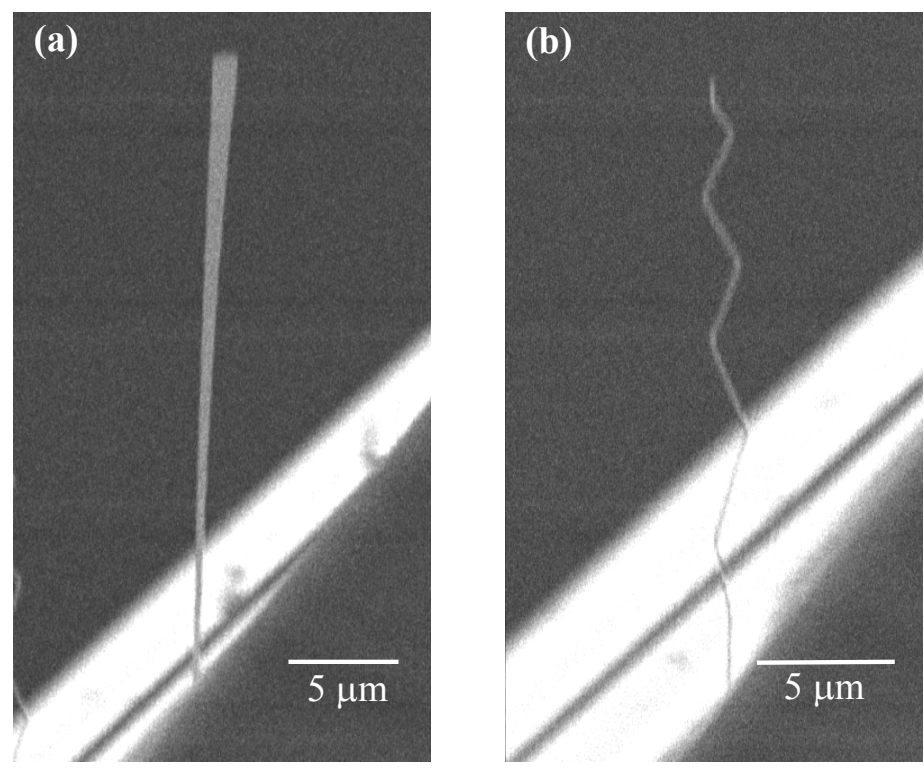

Fig. 9 Two types of resonators fabricated by FIB-CVD with one-dimensional FIB multiscan

(a) Long thin plate broadening to its end

(b) Zigzagging pillar

Table 2. Comparison of multi-layer model and single-layer model for each resonator

\begin{tabular}{cccc}
\hline \hline & $\begin{array}{c}\text { EB measurement } \\
{[\mathrm{kHz}]}\end{array}$ & $\begin{array}{c}\text { Multi-layer model } \\
{[\mathrm{kHz}]}\end{array}$ & $\begin{array}{c}\text { Single-layer model } \\
{[\mathrm{kHz}]}\end{array}$ \\
\hline Resonator (a) & 454 & $545(20.1 \%)$ & $631(39.0 \%)$ \\
Resonator (b) & 358 & $263(-26.6 \%)$ & $574(60.3 \%)$ \\
\hline \hline
\end{tabular}




\section{Conclusion}

We demonstrated that the analytical accuracy of FEM-based dynamic characteristic evaluation can be improved by using our proposed multi-layer analysis model. To design the dynamic characteristics of arbitrary 3D nanostructures fabricated by FIB-CVD, it is effective to take account of the distribution of the material properties in the structure, as shown in this paper. To further improve the accuracy, it is effective to improve the measurement precision of the material properties and to clarify the fabrication condition to determine the monostructure properties.

\section{Acknowledgments}

This work was partly supported by JSPS Grants-in-Aid for Scientific Research (1206016) and also by MEXT Nanotechnology Support Project (nanonet).

\section{References}

(1) Matsui, S., Kaito, T., Fujita, J., Komuro, M., Kanda, K., and Haruyama, Y., 2000, J. Vac. Sci. Technol. B, Vol. 18, 3181.

(2) Fujita, J., Ishida, M., Ichihashi, T., Sakamoto, T., Ochiai, Y., Kaito, T., and Matsui, S., 2002, Jpn. J. Appl. Phys. B, Vol. 41, No. 6, pp. 4423-4426.

(3) Kometani, R., Ishihashi, T., Kanda, K., Suzuki, T., Nihara, K., Ishihara, S., Kaito, T., and Matsui, S., 2008, J. Vac. Sci. Technol. B, Vol. 26, No. 6, pp. 2628-2631.

(4) Buks, E. and Roukes, M. L., 2001, Phys. Rev. B, 63033402.

(5) Utke, I., Hoffmann, P., and Melngailis, J., 2008, J. Vac. Sci. Technol. B, Vol. 26, No. 4, $1197-1276$.

(6) Website: http://www.srim.org

(7) Fujita, J., Okada, S., Ueki, R., Ishida, M., Kaito, T., and Matsui, S., 2007, Jpn. J. Appl. Phys. B, Vol. 46, No. 9, pp. 6286-6289.

(8) Igaki, J., Nakamatsu, K., Kometani, R., Kanda, K., Haruyama, Y., Kaito, T., and Matsui, S., 2006, J. Vac. Sci. Technol. B, Vol. 24, No. 6, pp. 2911-2914. 